\title{
CREATION OF DIGITAL TWINS - KEY CHARACTERISTICS OF PHYSICAL TO VIRTUAL TWINNING IN MECHATRONIC PRODUCT DEVELOPMENT
}

\author{
Sturm, Carolin; \\ Steck, Michael; \\ Bremer, Frank; \\ Revfi, Sven; \\ Nelius, Thomas; \\ Gwosch, Thomas; \\ Albers, Albert; \\ Matthiesen, Sven \\ IPEK Institute of Product Engineering, Karlsruhe Institute of Technology (KIT)
}

\begin{abstract}
Due to the falling costs of computational resources and the increasing potential of data acquisition, interest in digital twins, a virtual copy of the physical original, and their industrial application is increasing. Nevertheless, there is limited published work on how to support the process of physical to virtual twinning and what its key aspects are. The aim of this study is to present insights with regards to physical to virtual twinning gained from modelling projects in mechatronic product development. We conducted a survey and in-depth interviews with members of modelling projects. In the surveys and interviews we identified how physical products and virtual models were linked, which virtual models were used and which general challenges and key aspects are considered important by the project members. Our findings show that the key characteristics that pose challenges to modelling regarding physical to virtual twinning are model granularity, model validation, and model integration and interconnectivity.
\end{abstract}

Keywords: Digital Twin, Product modelling / models, Simulation, Design process

\section{Contact:}

Sturm, Carolin

Karlsruhe Institute of Technology (KIT)

IPEK Institute of Product Engineering

Germany

carolin.sturm@kit.edu

Cite this article: Sturm, C., Steck, M., Bremer, F., Revfi, S., Nelius, T., Gwosch, T., Albers, A., Matthiesen, S. (2021) 'Creation of Digital Twins - Key Characteristics of Physical to Virtual Twinning in Mechatronic Product Development', in Proceedings of the International Conference on Engineering Design (ICED21), Gothenburg, Sweden, 16-20 August 2021. DOI:10.1017/pds.2021.78 


\section{INTRODUCTION}

The important role of models in engineering is well known and accepted throughout the field. Especially in mechanical design, engineering teams use many different models to support the product development process (Matthiesen et al., 2019; Matthiesen et al., 2018; Grauberger et al., 2020). An important aspect especially in mechanical design is modelling the relationship between the embodiment and the behaviour of a product. Although modelling is essential for design engineering, the transition between physical, virtual and mental models is still a research topic (Jones et al., 2020b). With the increasing availability of field data and availability of computational power, it becomes more important to understand this transition between models. How the process of physical to virtual twinning can be supported and what key aspects are involved has been little researched so far.

In this contribution, we investigate insights about the process of physical to virtual twinning. We acquired the research data through a questionnaire survey with project members of product development projects. Aim of the questionnaire survey is to identify initial characteristics that support physical to virtual twinning. After data evaluation of the survey, we conducted interviews with selected project members for more detailed information. The interviews are designed to capture further details of digital twin characteristics and the underlying model building processes. The overall aim of this study is to identify key characteristics of modelling for physical to virtual twinning in mechatronic product development.

\subsection{Digital Twins and the twinning process}

Digital Twins enable engineers to simulate the behavior of physical products based on field data for continuous improvement.

\subsubsection{Definitions of the Digital Twin}

Many different definitions of a digital twin exist in literature (Jones et al., 2020a). Probably, NASA gave the first definition of it in their integrated technology roadmap (Mike Shafto et al., 2010). In most definitions, a digital twin consists of three main parts: physical product, virtual model, and connected data that tie and indissolubly connect the physical and virtual model (Schleich et al., 2017; Boschert and Rosen, 2016; Rosen et al., 2015; Glaessgen and Stargel; Grieves and Vickers, 2017). This interrelation is also shown in Figure 1.

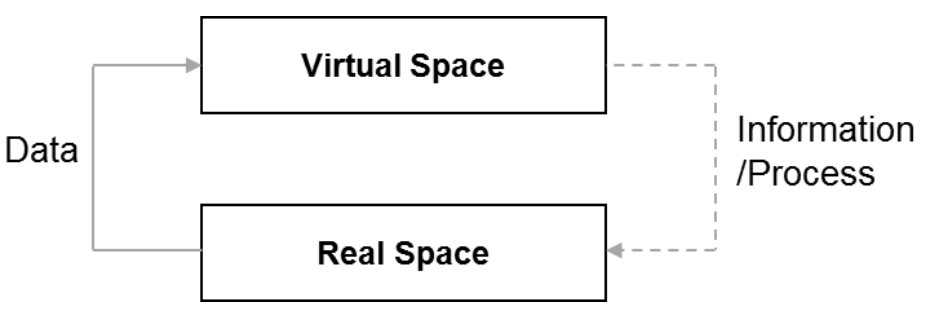

Figure 1. Twinning between the physical and virtual entities (Jones et al., 2020a)

The context of a digital twin can be manifold for example finance or engineering. This contribution focusses on the engineering context. Therefore, a distinction can be made between product, production and digital operations and service twin (Strietzel and Wagner, 2020). In the context of product development, Stark et al. (2020) define the digital twin as: "A digital twin is a digital representation of a product instance (real device, object, machine, service or intangible good) or an instance of a product-service system (a system consisting of a product and an associated service). Likewise within this digital representation during different life cycle phases different models, information and data are linked together."

A digital twin in engineering can be used, for example, to diagnose and predict real product instance behavior, to analyse changed operating conditions based on simulation models, and to ensure reliable new product generations. In product engineering are differences whether the digital twin is already used for the conceptual design and detail design phase of the product or only with regard to 
production. (Wagner et al., 2019; Haefner and Lanza, 2017; Riedelsheimer et al., 2020) Depending on the purpose of the digital twin, an adapted design of the digital twin is required.

\subsubsection{Virtual model and physical product}

A digital twin instance is derived from a digital master or generated from a physical product instance. The digital twin instance should be created at the beginning of the product development phase and continuously developed with focus to improve functionality. (Jones et al., 2020a) It is the exact description of the respective product instance. Nowadays, data is even more accessible, since low-cost sensors can be integrated into products and evaluated via the internet. From this, new insights for product development can be derived by physical to virtual twinning. The physical to virtual connections (twinning) describe the comparison between physical product and virtual model, the use of gained data and transfer of derived insights between physical product and digital twin. New insights can include, for example, real-time error detection in remote monitoring, especially when the products are difficult to access. The continuous observation of the behavior of a real product using its digital twin enables the detection of behavioural anomalies and the initiation of reactive measures. But, context knowledge from the development is necessary to interpret the operating data correctly during physical to virtual twinning. (Schleich et al., 2018; Söderberg et al., 2017; Söderberg et al., 2018)

\subsection{Digital Twin in engineering}

The various existing concepts of the digital twin also differ in the intended use of the digital twin during the product life cycle. In product engineering data from many previous product generations can be merged into a digital twin of the latest product generation to gain more knowledge in early phases of a new development cycle (Tao et al., 2018). Data from digital twins of previous product generations can be analysed and used to optimize new designs. In this regard, the application of digital twins can provide a design tool for quantitative design decisions in early phases. The recognition and feedback of usage and behavioural scenarios of the real product via the digital twin into the product development enables the definition of functional requirements beyond the acquisition of pure load data for dimensioning. Usage and behavior of the real product in the usage phase are important information for the determination of the requirements and the development of new product generations. It also enables estimation of the properties of future product generation life cycles. Practical examples from the creation of a digital twins and experiences which kind of modelling is necessary are little published in the product development section. There is limited knowledge about key aspects of modelling for physical to virtual twinning when considering the use of digital twins in mechatronic product development. With the following investigation, we would like to contribute to closing this gap.

\section{MATERIALS AND METHODS}

We conducted a study on characteristics of modelling the behaviour of technical systems, in particular the relation between embodiment and behaviour, to gain insights on physical to virtual twinning. The study consisted of a questionnaire and an interview. Product developer who could be involved in model building were contacted. The questionnaire was used to identify projects where a digital twin was used. Project members that indicated physical to virtual twinning were then additionally interviewed. The interviews were designed to identify detailed characteristics of modelling that could support the process of physical to virtual twinning. The following sections introduce the projects selected for the study, the questionnaire design, the key questions of the interviews and how the data was evaluated.

\subsection{Projects selected for the study}

The focus lay on projects in product development that deal with the relationship between embodiment and behaviour. In order to gain an insight into processes and characteristics of physical to virtual twinning, projects with both physical systems and virtual models were analysed. Project members were asked to participate in the survey. If the projects indicated physical to virtual twinning, they were analyzed in more detail in a second stage by interview.

\subsection{Questionnaire design}

The questionnaire had six sections and aimed to identify initial characteristics that support physical to virtual twinning. In a systematic literature review, Jones (2020) outlines the characteristics of digital 
twins (Jones 2020). Our questionnaire addressed characteristics as presented by Jones and was supplemented by questions about the projects, and possible challenges and potential support for physical to virtual twinning. Table 1 shows the structure of the questionnaire.

Table 1. Structure of the questionnaire

\begin{tabular}{|c|c|c|}
\hline Sections of the questionnaire & Content of the sections & $\begin{array}{l}\text { Investigated characteristics } \\
\text { according to Jones }(\mathbf{2 0 2 0})\end{array}$ \\
\hline Situation analysis & - $\quad$ Project key data & \\
\hline Problem containment & 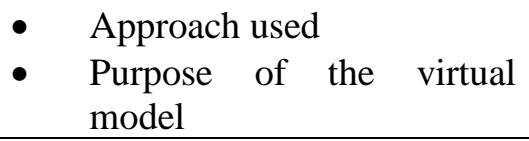 & \\
\hline Structure of the virtual model & - $\quad$ Types of models used & $\begin{array}{ll}\text { - } & \text { Virtual Entity/Twin } \\
\text { - } & \text { Virtual Environment } \\
\end{array}$ \\
\hline Physical product & - $\quad$ Measured variables & - $\quad$ Metrology \\
\hline $\begin{array}{l}\text { Links between virtual model and } \\
\text { physical product }\end{array}$ & $\begin{array}{ll}- & \text { Existence of links } \\
- & \text { Real-time exchange of data }\end{array}$ & $\begin{array}{ll}- & \text { Physical-to-Virtual } \\
& \text { Connection } \\
\text { - } & \text { Virtual-to-Physical } \\
\text { Connection }\end{array}$ \\
\hline Challenges and potential support & $\begin{array}{l}\text { - Challenges } \\
\text { - Aspects that should be } \\
\text { considered }\end{array}$ & \\
\hline
\end{tabular}

In the situation analysis section the project key data, like project duration, number of team members and the type of project is specified. The problem containment section is divided into two parts: the approach used and the purpose of the virtual model. The approach used could be entered as a free text. The purposes of the modelling according to Andreasen et al. (2015) can be checked in checkboxes and further purposes can be added. The purposes capture the unknown, define the design, obtain insight, manage, communicate, maintenance, predict behaviour and verification were given as options and examples for clarification. In the structure of the virtual model section, different types of models can be selected with checkboxes, for example structural model, thermic model and electromagnetic model. In order to investigate the characteristics according to John's (2020a) Metrology in the physical product, the various variables measured in the system could be ticked in checkboxes in the questionnaire. Examples for possible variables in the questionnaire are temperature, torque and pressure. In the Link between virtual model and physical product section, free text fields were used to check if there is a link between virtual and physical product and if real-time data transmission is implemented. In the last section of the questionnaire, free text fields were used to ask for challenges and aspects that should be considered when implementing a digital twin. Based on the questionnaire, we identified projects with a linking of the physical system and the virtual models for further investigation within the interviews.

\subsection{Interview study}

Projects that indicated physical to virtual twinning in the survey were examined in more detail in interviews with project members. Based on the questionnaire results, the interviews were designed to gain further information about characteristics defined by Jones et al. (2020a). Projects with linking between the physical product and the virtual model were selected for the interviews.

Table 2. Structure of the semi-structured interviews

\begin{tabular}{l|ll}
\hline Topic addressed in the interview & $\begin{array}{l}\text { Investigated characteristics according to Jones } \\
(2020)\end{array}$ \\
\hline Purpose of the digital twin & & \\
\hline$-\quad$ Contents of the digital master & $\bullet$ & Virtual Environment \\
& $\bullet$ & Virtual Processes \\
\hline - Model type used in the digital twin & $\bullet$ & Virtual Environment \\
- $\begin{array}{l}\text { Linking of the models in the digital twin } \\
\text { - Input and output between the digital } \\
\text { models }\end{array}$ & $\bullet$ & Virtual Processes \\
\hline
\end{tabular}




\begin{tabular}{|c|c|}
\hline - $\quad$ Input and output of the digital twin & \\
\hline $\begin{array}{l}\text { - } \quad \text { Type and structure of the physical product } \\
\text { - } \quad \text { Input and output of the physical product }\end{array}$ & $\begin{array}{l}\text { - Physical Environment } \\
\text { - Physical Processes } \\
\text { - State }\end{array}$ \\
\hline $\begin{array}{l}\text { - Linking of the virtual model and the } \\
\text { physical product } \\
\text { - Input and output of the virtual model and } \\
\text { the physical product } \\
\text { - Purpose of the link }\end{array}$ & $\begin{array}{ll}\text { - } & \text { Twinning } \\
\text { - } & \text { Physical-to-Virtual Connection/Twinning } \\
\text { - } & \text { Virtual-to-Physical Connection/Twinning } \\
\text { - } & \text { State }\end{array}$ \\
\hline $\begin{array}{l}\text { Comparison between physical product and } \\
\text { virtual model behavior }\end{array}$ & \\
\hline
\end{tabular}

Together with the interviewees and based on the addressed topics, process diagrams were developed in the interviews following the template of the diagrams shown in Figure 2. The diagrams illustrate different characteristics and facilitate, through visualization, the development of a common understanding.

\subsection{Data evaluation}

The data generated in the questionnaire and the interview study were evaluated as follows. The quantitative data collected in the questionnaire is used to cluster the projects according to their purposes and to evaluate the used virtual models. The qualitative data in the questionnaire was compiled by the authors and clustered with regard to different statements. The process diagrams created in the interviews were evaluated and compared with regard to the characteristics identified by Jones (2020a) in Table 2. The characteristics were compared with the stated purposes according to Andreasen (2015). Similarities and remarkable characteristics were analysed qualitatively.

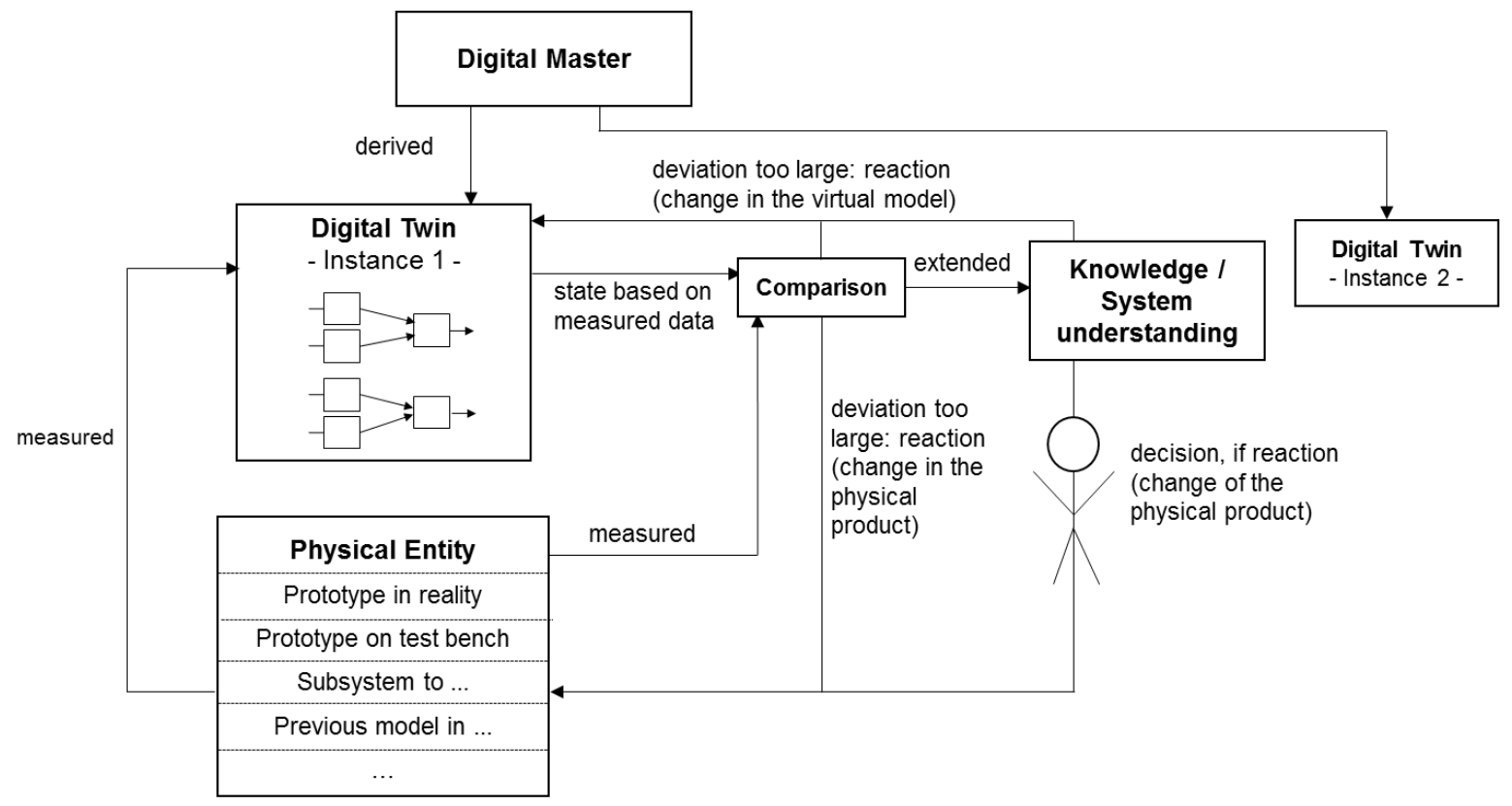

Figure 2. Template of the process diagram used in the interviews

\section{INVESTIGATION RESULTS}

From 104 project members and managers that we contacted, 13 returned the questionnaire. Only persons from the addressed group who actually deal with a combination of virtual model and physical product sent back the questionnaire. All contacted persons were from the industrial and research network of the authors. The participants of the questionnaire are mechanical engineers with projects in automotive, aircraft and power-tool development. One incomplete questionnaire was returned, which resulted in 12 full datasets. 9 of the projects were carried out as part of joint research and 3 were bilateral projects with industrial participants. The project duration ranged from three to 39 months (mean value 
26.75 months, standard deviation 10.8). One to three team members worked in the projects (mean value 1.8, standard deviation 0.7 ). 7 of the 12 projects indicated a link between the physical product and the virtual models in the questionnaires and were therefore examined in more detail in interviews. All projects are part of research projects. They are representative for early stages in product development. Therefore, the physical product is limited to prototypes and test bench installations.

Within the projects, virtual entities were created for different purposes (see Figure 3). The questionnaire to identify initial aspects shows the distribution of purposes of the projects considered.

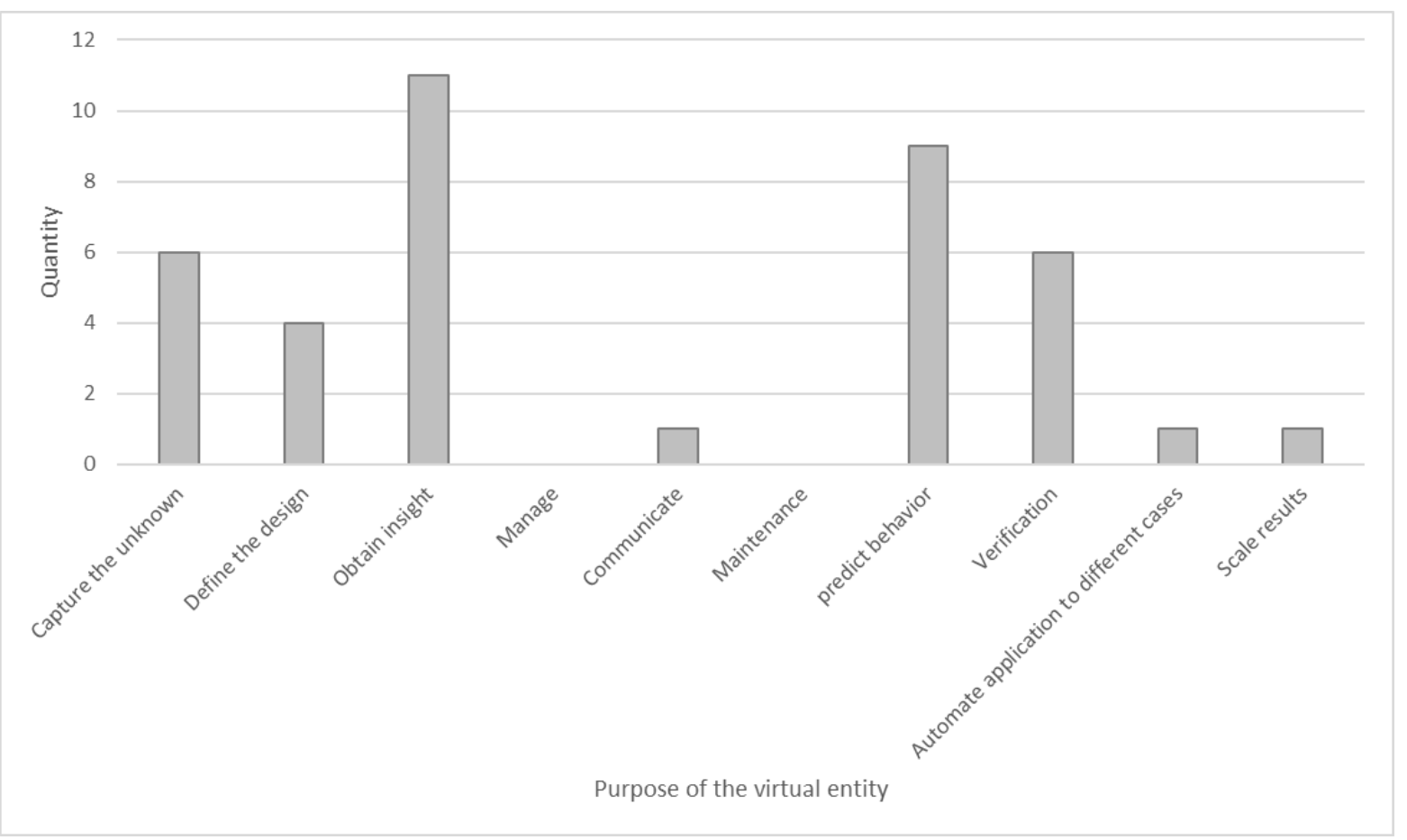

Figure 3. Virtual model purposes stated in the questionnaire

The most frequent purpose of the projects was Obtain insight (11 mentions), the second most frequent was Predict behaviour (9 mentions). The following purposes were also frequently addressed in the projects: Capture the unknown (6 mentions) and Verification (6 mentions). Less often, the purpose Define the design was chosen. The virtual environment was built with different purposes according to Andreasen et al. (2015). In the following section, correlations between different purposes, captured in the questionnaire and the expression of the characteristics, analysed in the interviews, are evaluated. Wide varieties of digital models are used. Most frequently Structural Model was used (8 mentions). The representation of the geometry was very often done with CAD models (6 mentions). Fluid dynamic models were also frequently used (4 mentions). Types of models like Multibody, Thermic model and control model were also mentioned several times and used in several projects. In individual projects also Electromagnetic model, MBSE and specialized Model for simulations of traffic were used.

In 6 of 7 projects which were examined in more detail in the interviews, Obtain insight was stated as a purpose. Communicate was only mentioned as a purpose in one project. Capture the unknown, predict behavior, define the design and verification were mentioned in 2-5 projects. As part of the projects, the virtual models used were also examined. In the interviews, one to four models were named in the virtual area, which were used and partly linked with each other in one project. Only one of the evaluated projects used Model Center, a software for integration of multiple modelling tools and collaboration between team members, as the central interface between the different virtual models and the physical product. In the other projects where models were linked, models were linked directly. Projects where capture the unknown was the purpose use three to four models in the virtual environment. The other projects use one or two models. Three projects had the purpose to define the design. These were the only projects where the physical system was changed due to a comparison between the digital twin and the physical product. 


\subsection{Modelling and validation of the models}

Five of the projects examined in the questionnaire work with a physical product and virtual models but did not directly link them. In all projects the relationship between embodiment and behaviour was modelled in the virtual models. Data, such as geometry or measured characteristics, from the physical product were used for the initial parameterization of the virtual instances/models. The virtual models were then iteratively developed further in part of the projects. In two of the projects the behaviour of the physical product was compared with the simulated behavior of the digital twin and was used to parameterize and verify the virtual model. If the behaviour did not sufficiently match, the virtual model was adapted in both cases. This was an iterative process which also led to a changing level of detail for the virtual models. In two of the projects, the output from the digital twin was compared with requirements for the product and used to determine whether the product should be developed further. The comparison took place regularly. Two projects use the comparison between the digital twin and the physical product to build up system understanding. Here, too, the comparison took place regularly.

\subsection{Physical to virtual connection}

For modelling purposes and for interaction between physical product and virtual model measurement data were collected. A differentiation between three types of data can be made. First, data about the embodiment, both properties and characteristics, as dimensions, weight, frictional coefficients, or stiffness. These data are used to improve the virtual representation of the physical product. Second, data representing the behaviour of the product. Third, environmental parameters that influence product behaviour. Data representing behaviour were mainly torque, rotational speed, force curvature, voltage, and current. The most frequent environmental parameter influencing the behaviour was temperature. The definition of the interfaces and thus their measured variables, which are transferred between physical product and virtual model, was called key aspect in the questionnaire.

\subsection{General challenges and key aspects}

The questionnaire asked about challenges in the projects. Four challenges were named with regard to physical to virtual twinning:

- Detailing of the models (2 mentions)

- Dealing with differences between digital twin and physical system (1 mention)

- $\quad$ Managing different setup states (1 mention)

- Keep the simulation time manageable (1 mention)

When asked which aspects should be considered in general when implementing digital twins, the following aspects were mentioned:

- $\quad$ Define the purpose (3 mentions)

- Define interfaces between digital twin and physical system (2 mentions)

- Simulation often needs individual input data, which is not available for the considered system (1 mention)

- $\quad$ Simplification (1 mention)

- $\quad$ Model updating (1 mention)

\section{DIscussion}

The main purpose of the modelling activities are aimed at gaining new insights about the system, obtain insight and capture the unknown. This also comes along with the purpose of predicting behaviour for which a sound understanding of the product is necessary.

\subsection{General modelling of embodiment-behaviour relations}

Out of the returned questionnaires, almost half of the projects only planned on connecting the physical product with the virtual model. These projects were aimed at gaining new insights on details in subsystems of the system under development. The results are used for dimensioning or improving embodiment of products. Such projects are still relevant for building digital twins. All projects without the explicit aim of building a digital twin aimed at increasing the understanding of a system through research with modelling and validation activities. Physical to virtual twinning for creating a digital 
twin requires detailed knowledge about the systems behaviour. Since the projects explicitly aim at creating models of the physical products it is generally possible to integrate this knowledge in a digital twin of the product or a future product generation. The key aspect of modelling the relationship between embodiment and behaviour is therefore the explicit documentation of knowledge. The elements built up in these projects can be integrated during physical to virtual twinning of a product and improve the overall modelling quality or the quality of predictions by the digital twin. These projects could also be seen as initial feasibility studies for a digital twin implementation.

\subsection{Model granularity}

The specification of the needed levels of detail was mentioned in the questionnaire both under the challenges and the aspects that should be considered in general when implementing digital twins. The iterative validation of the models and the mention that it is challenging to define the purpose of the model further support this. Model granularity typically changes during validation as well as due to a changing purpose of the model. This is a well-known challenge for modelling in general and is for example described by Maier et al. (2017). The mentions in the interviews from projects with digital twin support that this is a challenge for twinning as well.

\subsection{Model validation}

The more detailed analysis of the projects which stated that they have implemented a linking between the physical product and virtual model in the interviews showed different stages of application and understanding of the digital twin. Validated models, for example FE-models or other structural models are used for design and dimensioning of the physical product. In most cases, the physical system was especially designed for gaining insight in the behaviour. The most frequent use of the digital twin contained in the projects are models for initial dimensioning. The validation conducted afterwards then resulted in an increased knowledge about the system which was carried on to the next generation of virtual and physical instances. The key aspect we identified here is creating a valid model during physical to virtual twinning. This requires a direct or indirect link through measurable values to evaluate the capacity of the model to predict the system behaviour based on the embodiment parameters, or modelling quality. When using applicable state of the art models, the projects revealed that the validation does not necessarily need to be conducted on a detailed level but can start on a higher level. Therefore, using existing models of the relationship between embodiment and behaviour could improve twinning because validation steps have already been done on a detailed level by researchers for the individual models.

\subsection{Model integration and interconnectivity}

Although it is a core element of the concept of a digital twin, another key aspect of physical to virtual twinning is identifying measurable values for linking virtual and physical product. This was mainly reported as a challenge due to increasing complexity of both entities when integrating multiple sensors in the physical product as well as processing them in a meaningful way in the virtual model. As seen in the answers on what to consider in general when implementing a digital twin.

To summarize, modelling subsystem behaviour in general without building a digital twin can provide useful insights to apply in later attempts of building a digital twin. Besides this, the identified key characteristics of modelling the relation of embodiment and behaviour for physical to virtual twinning are:

- Model granularity - Selection of the detail level of the model

- Model validation - Efficient validation of models

- Model Integration and Interconnectivity - Identification of measurable values for linking virtual and physical product / virtual and digital twin

\section{CONCLUSION}

In this contribution, we conducted a study on the physical to virtual twinning. We find that the key characteristics posing challenges in model building that apply to physical to virtual twinning are model granularity, model validation, and model integration and interconnectivity. Additionally, the questionnaire and interviews showed that modelling of physical behavior in general is relevant for physical to virtual twinning. We identified these characteristics through a questionnaire and additional 
interviews with members of modelling projects. The aspects show that modelling the relationship between embodiment and behaviour is part of physical to virtual twinning. Future research should focus on integrating knowledge on challenges and key characteristics of classical embodimentbehaviour modelling to the twinning process in research and industrial application. Therefore, more detailed work on the aspects of physical to virtual twinning should be conducted in focused single case studies to ensure a sufficient complexity and to allow an investigation of the twinning process beyond early phases.

\section{ACKNOWLEDGMENT}

The project upon which this publication is based was partly funded by Federal Ministry for Economic Affairs and Energy under the funding number 20Y1910E. The authors of this publication are responsible for its contents.

\section{REFERENCES}

Andreasen, M., Hansen, C. and Cash, P. (2015), Conceptual Design, Springer, Cham Heidelberg New York Dordrecht London.

Boschert, S. and Rosen, R. (2016), "Digital Twin-The Simulation Aspect", in Hehenberger, P. and Bradley, D. (Eds.), Mechatronic Futures, Springer International Publishing, Cham, pp. 59-74.

Glaessgen, E. and Stargel, D., "The Digital Twin Paradigm for Future NASA and U.S. Air Force Vehicles", in 53rd AIAA/ASME/ASCE/AHS/ASC Structures.

Grauberger, P., Bremer, F., Sturm, C., Hoelz, K., Wessels, H., Gwosch, T., Wagner, R., Lanza, G., Albers, A. and Matthiesen, S. (2020), "Qualitative Modelling In Embodiment Design - Investigating the Contact and Channel Approach Through Analysis of Projects", Proceedings of the Design Society: DESIGN Conference, Vol. 1, pp. 897-906.

Grieves, M. and Vickers, J. (2017), “Digital Twin: Mitigating Unpredictable, Undesirable Emergent Behavior in Complex Systems", in Kahlen, F.-J., Flumerfelt, S. and Alves, A. (Eds.), Transdisciplinary Perspectives on Complex Systems, Vol. 89, Springer International Publishing, Cham, pp. 85-113.

Haefner, B. and Lanza, G. (2017), "Function-oriented measurements and uncertainty evaluation of micro-gears for lifetime prognosis", CIRP Annals, Vol. 66 No. 1, pp. 475-478.

Jones, D., Snider, C., Nassehi, A., Yon, J. and Hicks, B. (2020a), "Characterising the Digital Twin: A systematic literature review”, CIRP Journal of Manufacturing Science and Technology, Vol. 29, pp. 36-52.

Jones, D.E., Snider, C. and Hicks, B. (2020b), “A Framing of Design as Pathways Between Physical, Virtual and Cognitive Models", Proceedings of the Design Society: DESIGN Conference, Vol. 1, pp. 41-50.

Maier, J.F., Eckert, C.M. and John Clarkson, P. (2017), "Model granularity in engineering design - concepts and framework", Design Science, Vol. 3, p. 1.

Matthiesen, S., Grauberger, P., Bremer, F. and Nowoseltschenko, K. (2019), "Product models in embodiment design: an investigation of challenges and opportunities", SN Applied Sciences, Vol. 1 No. 9, p. 423.

Matthiesen, S., Grauberger, P., Sturm, C. and Steck, M. (2018), "From Reality to Simulation - Using the C\&C2Approach to Support the Modelling of a Dynamic System”, Procedia CIRP, pp. 475-480.

Mike Shafto, Mike Conroy, Rich Doyle, Ed Glaessgen, Chris Kemp, Jacqueline LeMoigne and Lui Wang (2010), "DRAFT Modeling, Simulation, information Technology \& Processing Roadmap", NASA Modeling, Simulation, Information Technology \& Processing - TA11.

Riedelsheimer, T., Lünnemann, P., Wehking, S. and Dorfhuber, L. (2020), Digital Twin Readiness Assessment: Eine Studie zum Digitalen Zwilling in der Fertigenden Industrie.

Rosen, R., Wichert, G. von, Lo, G. and Bettenhausen, K.D. (2015), “About The Importance of Autonomy and Digital Twins for the Future of Manufacturing”, IFAC-PapersOnLine, Vol. 48 No. 3, pp. 567-572.

Schleich, B., Anwer, N., Mathieu, L. and Wartzack, S. (2017), "Shaping the digital twin for design and production engineering”, CIRP Annals, Vol. 66 No. 1, pp. 141-144.

Schleich, B., Wärmefjord, K., Söderberg, R. and Wartzack, S. (2018), “Geometrical Variations Management 4.0: towards next Generation Geometry Assurance”, Procedia CIRP, Vol. 75, pp. 3-10.

Söderberg, R., Wärmefjord, K., Carlson, J.S. and Lindkvist, L. (2017), “Toward a Digital Twin for real-time geometry assurance in individualized production”, CIRP Annals, Vol. 66 No. 1, pp. 137-140.

Söderberg, R., Wärmefjord, K., Madrid, J., Lorin, S., Forslund, A. and Lindkvist, L. (2018), “An information and simulation framework for increased quality in welded components", CIRP Annals, Vol. 67 No. 1, pp. 165-168.

Stark, R., Anderl, R., Thoben, K.-D. and Wartzack, S. (2020), “WiGeP-Positionspapier: „Digitaler Zwilling“", ZWF Zeitschrift für wirtschaftlichen Fabrikbetrieb, Vol. 115 No. special, pp. 47-50.

Strietzel, M. and Wagner, L. (2020), "Herausforderung Digital Twin: Die digitale Durchgängigkeit erfolgreich gestalten”, NAFEMS DACH Proceedings 2020. 
Tao, F., Cheng, J., Qi, Q., Zhang, M., Zhang, H. and Sui, F. (2018), "Digital twin-driven product design, manufacturing and service with big data", The International Journal of Advanced Manufacturing Technology, Vol. 94 No. 9-12, pp. 3563-3576.

Wagner, R., Schleich, B., Haefner, B., Kuhnle, A., Wartzack, S. and Lanza, G. (2019), "Challenges and Potentials of Digital Twins and Industry 4.0 in Product Design and Production for High Performance Products", Procedia CIRP, Vol. 84, pp. 88-93. 